\title{
Effects of intermittent versus continuous dieting on weight and body composition in obese and overweight people: a systematic review and meta-analysis of randomized controlled trials
}

\section{(退 Article in press ? )}

- Roman, Y.M.a,

- Dominguez, M.C. ${ }^{a}$,

- Easow, T.M.a,

- Pasupuleti, V. ${ }^{\mathrm{b}}$,

- White, C.M. ${ }^{a}$,

- Hernandez, A.V.a,cemail Author

- View Correspondence (jump link)

- aHealth Outcomes, Policy, and Evidence Synthesis (HOPES) Group, School of Pharmacy, University of Connecticut, Storrs, CT, United States

- $\quad$ 'ProEd Communications, Inc, Cleveland, OH, United States

- School of Medicine, Universidad Peruana de Ciencias Aplicadas (UPC), Lima, Peru

\section{Abstract_View references (26)}

Background: Intermittent dieting may be an alternative to continuous dieting for weight reduction. Objective: To evaluate the effect of intermittent dieting versus continuous dieting on weight and body composition in overweight or obese adults. Design: A systematic review and meta-analysis of randomized controlled trials (RCTs). Five databases were searched until February 2018 for RCTs comparing intermittent versus continuous dieting. Intermittent dieting consisted of two types: regular intermittent was caloric restriction interspersed with days of weight maintenance or ad libitum eating; intensified intermittent was caloric restriction interspersed with days of even lower caloric restriction. Continuous was continual caloric restriction. Primary outcomes were weight, body fat, lean mass, waist circumference, hip circumference, and energy expenditure. Data were pooled by the inverse variance method using random-effects models and expressed as mean differences (MD) and their 95\% confidence intervals (Cl). Results: Nine trials met the inclusion criteria $(n=782)$, six comparing regular intermittent vs continuous ( $n=553$ ), and three comparing intensified intermittent vs continuous ( $n=229$ ). Populations were heterogeneous: obese only in five studies, and overweight or obese (mixed) in four studies. Lean mass was significantly lower in regular intermittent vs continuous (MD $-0.86 \mathrm{~kg} ; 95 \% \mathrm{Cl}$ -1.62 to $-0.10 ; p=0.03$ ). No differences were found for the remaining outcomes for both comparisons (regular intermittent or intensified intermittent vs continuous). There was low heterogeneity of effects across trials. Subgroup effects by time to follow-up, gender, per-protocol versus intention-to-treat, enforced exercise, and diabetes were similar to main analyses. Conclusions: This systematic review in obese and overweight individuals showed that regular intermittent dieting decreased lean mass compared to continuous dieting. There were no differences in effects for either intermittent vs continuous interventions 
across all other outcomes. In contrast to previous systematic reviews, this study suggested that lean mass is better preserved in continuous dieting compared to regular intermittent dieting. (C) 2018, Springer Nature Limited.

SciVal Topic Prominence

Topic: Fasting | Weight Loss | intermittent fasting Prominence percentile: 91.361

- ISSN: 03070565

- CODEN: IJOBD

- Source Type: Journal

- Original language: English

- DOI: $10.1038 / \mathrm{s} 41366-018-0204-0$

- Document Type: Article in Press

- Publisher: Nature Publishing Group 